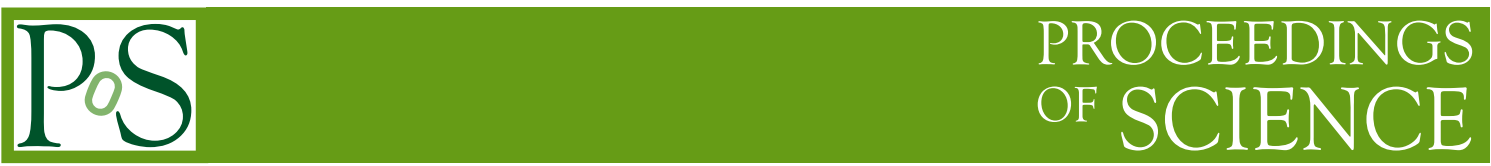

\title{
Rare Meson Decays and Transition Form Factors
}

\author{
Susan Schadmand* \\ Forschungszentrum Jülich \\ E-mail: s.schadmandefz-juelich.de
}

The WASA-at-COSY Collaboration

The physics program with the WASA detector at the COSY accelerator pursues symmetry breaking processes by studying rare decays of the light unflavored mesons as well as the determination of electro-magnetic transition form factors. The WASA facility is a $4 \pi$ detector system, designed to study the hadronic production and the decays of light mesons. A high density pellet target combined with the high intensity beams of the Cooler Synchrotron COSY provide luminosities which allow studies of rare processes.

Among the cases to be discussed are the three-pion decays of $\eta$ and $\omega$ mesons which hold important information for chiral perturbation theory. At low energies, the dynamics of pseudoscalar Goldstone bosons is successfully described by chiral perturbation theory. New strategies are being explored to describe processes at higher energies with additional degrees of freedom. Leadingorder calculations for two-body decays and decays of vector mesons to three pseudoscalar mesons are already available.

The study of the conversion decays of pseudoscalar and vector mesons aim at the precise determination of fundamental hadron properties, here, the transition form factors. The knowledge of form factors is needed for the interpretation of the $\mathrm{g}-2$ and $\pi^{0} \rightarrow e^{+} e^{-}$experiments. The case of the $\omega \rightarrow \pi^{0} e^{+} e^{-}$decay provides unique information about the form factor in the time-like region where the two vector particles (the $\omega$ and the intermediate virtual photon) have an invariant mass squared significantly greater than zero. The puzzling issue has been that the transition form factor for the $\omega$ meson seems to deviate strongly from vector meson dominance predictions whereas pseudoscalar meson decays involving dileptons typically agree with vector meson dominance, for example, $\pi^{0} \rightarrow \gamma e^{+} e^{-}$and $\eta \rightarrow \gamma \mu^{+} \mu^{-}$. Here, both theoretical and experimental advances have recently been made.

Sixth International Conference on Quarks and Nuclear Physics

April 16-20, 2012

Ecole Polytechnique, Palaiseau, Paris

${ }^{*}$ Speaker. 


\section{Meson Decay Physics}

The meson decay physics program with the WASA detector at the COSY accelerator of the Forschungszentrum Jülich, Germany, pursues symmetry breaking processes by studying rare decays of the light unflavored mesons as well as the determination of electro-magnetic transition form factors. The decay patterns of light mesons are exploited as a laboratory for the strong interaction. The physics objectives include symmetries and symmetry violation in rare decays, quark mass differences, test of theoretical predictions, and the determination of parameters for low energy QCD, such as the transition form factors. Finally, rare decays can provide hints for non-conventional effects beyond the standard model. The WASA program is embedded in the European network 'MesonNet - Meson Physics in Low-Energy QCD' [1].

In the following, the study of the decays $\eta \rightarrow 3 \pi$ and $\omega \rightarrow 3 \pi$ mesons which are used for the extraction of Dalitz plot parameters are discussed, see section 2. In section 3, we report on our efforts to determine transition form factors using the decays $\eta \rightarrow \gamma\left(^{*}\right) e^{+} e^{-}$and $\omega \rightarrow \pi e^{+} e^{-}$. It should be noted here that the decay $\eta \rightarrow e^{+} e^{-} e^{+} e^{-}$carries information about the double transition form factor and is being studied with the first goal of a branching ratio [2, 3].

Form factors also play a prominent role in the decay $\eta \rightarrow \pi^{+} \pi^{-} \gamma$. At the chiral limit of zero momentum and massless quarks this decay is determined by the box anomaly term of the WessZumino-Witten Lagrangian. We have performed an exclusive measurement where the analysis, performed by C.F. Redmer [2, 4], shows that the pion angular distribution is consistent with a relative p-wave of the two-pion system whereas the photon energy spectrum does not agree with the simplest gauge invariant matrix element. A parametrization of the data can be achieved by the additional inclusion of the empirical pion vector form factor multiplied by a first-order polynomial in the squared invariant mass of the $\pi \pi$ system.

\section{1 experimental approach and data analysis}

The WASA facility is a $4 \pi$ detector system, designed to study the hadronic production and the decays of light mesons with the decay products $\gamma, \pi, e^{ \pm}$. The unique high density pellet target combined with high intensity beams of the Cooler Synchrotron COSY, Jülich, provide luminosities which allow studies of rare processes ${ }^{1}$.

Two production reactions are used with WASA-at-COSY. The reaction $p+d \rightarrow{ }^{3} \mathrm{He}+$ meson introduces relatively less background from direct multi-pion background which does not stem from the meson decay. The meson is readily tagged by detecting the ${ }^{3} \mathrm{He}$ recoil particle which is possible on the trigger level. Due to the the form factor in this fusion reaction, cross sections for meson production are generally much smaller than in $p p$ scattering. The reaction $p+p \rightarrow p+p+$ meson is used to produce high statistics data sets, necessary for very rare decays, at the cost of substantial multi-pion background.

The data analysis makes use of the features of the WASA detector [5]. Charged particle tracking is achieved within the field of a super-conducting solenoid magnet at central angles and particle identification and reconstruction is available for all final state particles. Photons are detected with a closely-packed, highly granular $\mathrm{CsI}(\mathrm{Na})$ electromagnetic calorimeter at central angles. The

\footnotetext{
${ }^{1}$ http://www2.fz-juelich.de/ikp/wasa/
} 
forwardly emitted recoil particles are tracked and reconstructed with stacks of straw tubes and segmented plastic scintillator hodoscopes. The complete detection of all final state particles is helped by choosing the optimal beam energy (forward boost) with respect to geometric acceptance of the central and forward part of the detector. A missing mass analysis using the beam parameters and the recoil particles enables us to tag on the meson production. Meson decays are studied by the detection of all decay products in the central part of the detector. The analysis profits from the complete detection of the reaction products by employing kinematic conditions and fitting for background suppression and resolution precision. The remaining background from multi-pion production is subtracted using a polynomial fit controlled by the knowledge of the resulting signal line shape in the missing mass spectrum. The subtraction of background from unwanted decays of the meson has to be achieved with the help of simulations. Systematic effects are studied intensively and stem from selection cuts as well as from uncertainties in assumptions made in the simulations. The results of the data analysis are total and differential cross sections or upper limits, Dalitz plots, branching ratios, form factors, and other parameters including systematic uncertainties for the physics interpretation.

In most of our meson decay studies cases, the experimental data analysis is trained with the $p d \rightarrow{ }^{3} \mathrm{HeX}$ reaction, confronted with detailed simulations and then further developed for the $p+p$ data sets. Finally, it is important to realize that it is mandatory to compare the results with other current experimental efforts [1].

\section{Dalitz plot analysis}

The three-pion decays of $\eta$ mesons hold important information for chiral perturbation theory and the promise to extract limits on the quark mass differences. The decay width scales as $\Gamma=$ $\left(\frac{Q_{D}}{Q}\right)^{4} \bar{\Gamma}$ where $Q^{2}=\left(m_{s}^{2}-\hat{m}^{2}\right) /\left(m_{d}^{2}-m_{u}^{2}\right), \hat{m}=\frac{1}{2}\left(m_{u}+m_{d}\right)$. The decay width $\bar{\Gamma}$ and $Q_{D}=24.2$ are calculated in the Dashen limit [6]. $Q$ gives access to light quark mass ratios which serve as an important input for lattice QCD [7]. To derive $Q, \bar{\Gamma}$ has to be known reliably from theory which can be tested by comparing with the experimental Dalitz plot distributions.

For the case of $\eta \rightarrow 3 \pi^{0}$, we studied the Dalitz plot of the three pions where the quadratic slope parameter $\alpha$ was determined to be $-0.027 \pm 0.008$ (stat) \pm 0.005 (syst) [8]. The result has been consistent with previous measurements and further corroborates the importance of pion-pion final state interactions. Using our extended data set on $\eta$ decays as well as recently acquired data on $\omega$ decays, we continue the Dalitz plot analyses for light meson decays into $\pi^{0} \pi^{+} \pi^{-}$. Currently, the most precise experimental result for $\eta \rightarrow \pi^{0} \pi^{+} \pi^{-}$is based on a Dalitz plot containing $1.34 \cdot 10^{6}$ events obtained by KLOE [9]. Here, two second order parameters deviate significantly from chiral perturbation theory predictions. Independent measurements are important and we aim at providing two independent data sets with $\eta$ mesons produced in $p p$ and $p d$ reactions.

In [3], the preliminary acceptance-corrected experimental results with statistical errors for the projections of the Dalitz plot are presented. $10^{7}$ events from the $p d \rightarrow{ }^{3} \mathrm{He} \eta$ reaction have been analyzed employing bin-wise background subtraction. This yields $2 \times 10^{5}$ events in the Dalitz plot. The projections are in reasonable agreement with the KLOE experimental result. This work on the $p d$ data has been continued in order to obtain the Dalitz plot parameters for $\eta \rightarrow \pi^{+} \pi^{-} \pi^{0}$ 
including the study of systematic effects. Preliminary results for the Dalitz plot parameters are pending. More data are available from beam times using the reaction $p p \rightarrow p p \eta$.

The decay of the $\omega$ meson into $\pi^{0} \pi^{+} \pi^{-}$is used for the investigation of the $\omega$ decay mechanism to three pions from a comparison of a high-statistics density distribution of the Dalitz plot with theoretical predictions. The decay mechanism was considered within the vector meson dominance model as proceeding via the $\rho \pi$ intermediate state [10]. More recent predictions are based on a counting scheme for flavor-SU(3) systems of Goldstone bosons and light vector mesons [11]. The importance of the $\pi \pi$ final state interaction is also considered [12]. However, the expected deviations from the Vector Meson Dominance model are small.

The WASA-at-COSY experiment has produced a significant amount of fully reconstructed $\omega \rightarrow 3 \pi$ events using $p+p$ and $p+d$ reactions. Initial analyses have been performed on both data sets [3]. This presentation showed the result of $15 \%$ of the $1.5 \mathrm{GeV} p+d$ data set, yielding ca $9000 \omega \rightarrow 3 \pi$ events, and all of the pilot run with $2.063 \mathrm{GeVV} p+p$ data, yielding ca. 5500 events. For this, the missing mass analysis, with preliminary decay channel selection, is performed. A clear peak corresponding to ${ }^{3} \mathrm{HeX}(3 \pi)$ is seen as the result of careful tuning of the reconstruction and calibration. For the $p p 3 \omega$ case at this stage, only a shoulder above (multi pion) background in $p+p$ is discerned. Here, the analysis challenges are the intense background from direct $3 \pi$ production and high energy protons which are not stopped in the forward detector. Both analyses are promising on the way to the desired high-statistics Dalitz plots.

\section{Dalitz decays of mesons}

Our motivation is to measure the electromagnetic transition form factor of the $\omega$ meson Dalitz decay $\omega \rightarrow \pi^{0} \mathrm{e}^{+} \mathrm{e}^{-}$. According to standard vector meson dominance, this decay occurs via a virtual $\rho$ meson giving rise to the observed form factor at the $\omega \pi$ transition. Experimentally, the form factor is determined by comparing the dilepton invariant mass spectrum with the point-like quantum electrodynamics prediction. The issue has been that the observed transition form factor for the $\omega$ meson does not agree with (even extended) vector meson dominance while other meson decays involving dileptons, like $\pi^{0} \rightarrow \gamma e^{+} e^{-}$and $\eta \rightarrow \gamma \mu^{+} \mu^{-}$are consistent, as described in the 1986 review by L.G.Landsberg [13]. There is much improved agreement when the decays of narrow light vector mesons into pseudoscalar mesons and dileptons are calculated to leading order by treating pseudoscalar and vector mesons on equal footing. These theoretical efforts can attempt to go beyond vector meson dominance in a systematic way [14, 15]. The $\omega \rightarrow \pi^{0} \gamma^{\star}$ and $\phi \rightarrow \pi^{0} \gamma^{\star}$ electromagnetic transition form factors based on dispersion theory are calculated in [16], relying on the previous dispersive analysis of the corresponding three-pion decays and the pion vector form factor [12]. Recently, the NA60 collaboration has corroborated the previous data [17, 18]. In order to investigate these important issues, experiments using different experimental approaches are needed. Here, the approach is to use elementary reactions and full reconstruction of the decaying meson. Note, that the case of the $\phi$ meson is addressed by KLOE, see the contribution to this conference, while the investigation of the $\omega \pi$ transition is underway at WASA-at-COSY.

For the status and preliminary results on transition form factors, we presented preliminary results for the $\eta$ transition form factor from the decays $\eta \rightarrow$ yee. The results are based on two independent analyses using two different production mechanisms [2]. We point out further steps 
towards our final result, as in analysis of the complete available data sets, systematic studies, finetune calibrations, re-tune kinematic fit, and bin-wise background subtraction.

$\omega$ decays are being analyzed using the $p d \rightarrow{ }^{3} \mathrm{He} \omega$ experiment and the $p p \omega$ pilot run, as mentioned above. As a first step towards the $\omega \pi$ transition form factor, we study the decay with a real photon, $\omega \rightarrow \pi \gamma$. Promising first analyses observe the $\omega$ peak in a missing mass analysis, inclusively and in the presence of the respective decay products [3]. Using the two sets of experiments with two different reaction mechanisms, we aim to compare the quality of the data between $p d$ and $p p$ reactions, in the sense of a feasibility and background study for the analysis of $\omega \rightarrow \pi^{0} e^{+} e^{-}$ decays.

\section{Summary}

In summary, we have accumulated $3 \times 10^{7} \eta$ mesons produced in $\mathrm{pd} \rightarrow{ }^{3} \mathrm{He} \eta$ and $5-10 \times$ $10^{8} \eta$ decays in $\mathrm{pp} \rightarrow \mathrm{pp} \eta$. The experimental goal of studying the very rare meson decays like $\eta \rightarrow e e$ motivated the amount of data taken. The current studies of $\omega$ decays focus on two pilot experiments and will develop the experimental and analysis methods to process a planned highstatistics experiment to take place before 2015. The goal is a precise determination of the $\omega \pi$ transition form factor.

\section{References}

[1] A network within the project 'Study of Strongly Interacting Matter' (Hadron Physics3), an Integrating Activity within the Seventh Framework Program of EU, http://www2.fz-juelich.de/ikp/mesonnet/.

[2] WASA-at-COSY Dissertation, http://www2. fz-juelich.de/ikp/wasa/theses/.

[3] Proceedings of the Second International PrimeNet Workshop, nucl-ex/1204.5509 (2012).

[4] WASA-at-COSY Collaboration, P. Adlarson et al., Phys.Lett. B707, 243 (2012), 1107.5277.

[5] CELSIUS/WASA, C. Bargholtz et al., Nucl. Instrum. Meth. A594, 339 (2008), 0803.2657.

[6] R. F. Dashen, Phys.Rev. 183, 1245 (1969).

[7] G. Colangelo et al., Eur.Phys.J. C71, 1695 (2011), 1011.4408.

[8] WASA-at-COSY Collaboration, C. Adolph et al., Phys.Lett. B677, 24 (2009), 0811.2763.

[9] KLOE Collaboration, F. Ambrosino et al., JHEP 0805, 006 (2008), 0801.2642.

[10] M. Gell-Mann, D. Sharp, and W. G. Wagner, Phys. Rev. Lett. 8, 261 (1962).

[11] S. Leupold and M. F. M. Lutz, Eur. Phys. J. A 39, 205 (2009), 0807.4686.

[12] F. Niecknig, B. Kubis, and S. P. Schneider, Eur.Phys.J. C72, 2014 (2012), 1203.2501.

[13] L. Landsberg, Phys.Rept. 128, 301 (1985).

[14] C. Terschluesen and S. Leupold, Phys. Lett. B691, 191 (2010), 1003.1030.

[15] S. Leupold and C. Terschlusen, (2012), 1206.2253.

[16] S. P. Schneider, B. Kubis, and F. Niecknig, (2012), 1206.3098.

[17] NA60, R. Arnaldi et al., Phys. Lett. B677, 260 (2009), 0902.2547.

[18] NA60 Collaboration, A. Uras, J.Phys.Conf.Ser. 270, 012038 (2011), 1108.0968. 